\title{
SYPHILIS SIMULATING AUTOIMMUNE DISEASE: CASE REPORT
}

\author{
Tatiana Melo Fernandes ${ }^{1, \star}$, Mariana D’Oliveira Bulhões da Costa ${ }^{1}$ \\ 1.Hospital Naval Marcílio Dias, Rio de Janeiro (RJ), Brazil. \\ *Corresponding author: tatianamelofernandes@gmail.com
}

\section{BACKGROUND}

Syphilis is a bacterial infection caused by Treponema pallidum and its main route of transmission is sexual. It is divided into primary, secondary and tertiary according to its clinical manifestations and evolution time. Lesions in the oral cavity are not frequent and may delay the diagnosis.

\section{CASE REPORT}

Female, 38 years old, reports for 3 months the onset of oral cavity lesions associated with mild Raynaud's phenomenon in the hands. Previous history of breast implant surgery 7 months ago, superficial thrombosis in right arm, two spontaneous abortions in the first trimester and diagnosis of hereditary thrombophilia by mutation of the MTHFR gene. She denied family history of autoimmune disease, but father with a recent episode of pulmonary thromboembolism. She had painless oral ulcers on the hard, soft palate, tongue and lower lip (Figs 1 and 2). Laboratory showed an increase in liver and canalicular enzymes, and investigation was started for autoimmune hepatitis and primary biliary cholangitis through magnetic resonance cholangiopancreatography, abdominal ultrasound with portal system Doppler, which were normal. The anti-HIV test using the Elisa method was positive, but this diagnosis was later ruled out by Western blot. Negative antiphospholipid antibodies, positive (antinuclear antibody) ANA 1:80 fine speckled pattern, serum complement and protein electrophoresis normal and positive VDRL. During the consultation, she admitted that her husband had penile lesions. A biopsy of the lesion on the lower lip was performed with microscopy, revealing a perivascular lymphoplasmacytic infiltrate pointing to the possible diagnosis of secondary syphilis and the FTA-ABS test was positive in high titers.

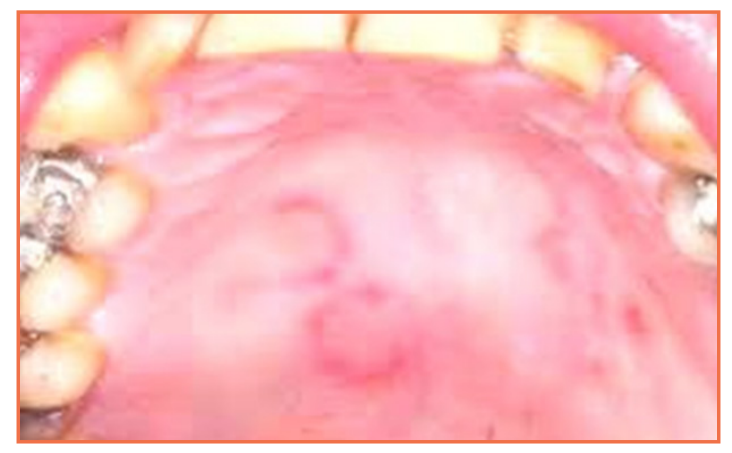

Figure 1. Ulcerated lesions in hard palate.

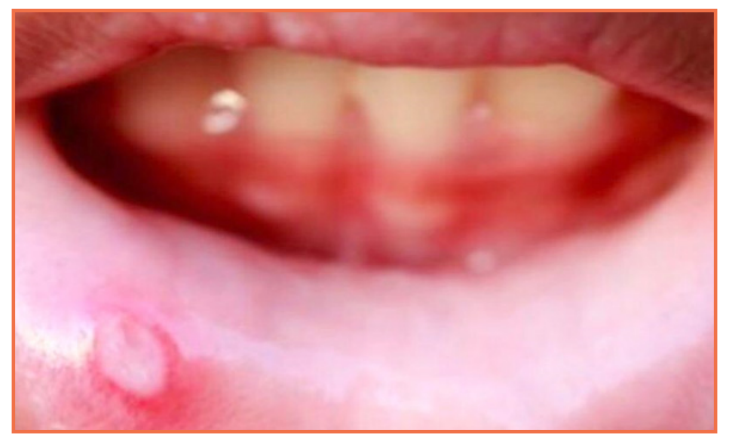

Figure 2. Solitary ulcerated lesion on the lower lip. 


\section{CONCLUSION}

The oral manifestations of syphilis are different depending on the stage of the disease. The secondary course leads to mucous lesions that are whitish, irregular and painless. The diagnosis of oral lesions can be made by direct visualization of the spirochetes in the biopsy tissue in addition to venereal disease research laboratory (VRDL), positive FTA-ABS in high blood titers and increased inflammatory tests in the serum. In addition, anatomopathology shows chronic mononuclear inflammation with a predominance of plasma cells, especially around blood vessels as described in our case. Our patient presented with secondary syphilis associated with previous superficial thrombosis in the right upper limb; however, her antiphospholipid antibodies were negative in several doses, concluding that it was an isolated infectious condition simulating an autoimmune disease. 